\title{
Empatia e Arte - Poderá um Programa de Arte numa Unidade de Saúde Familiar Influenciar a Empatia Percecionada pelos Utentes?
}

\author{
Empathy and Art - Can an Art-based Program in a Primary Care Unit \\ Influence Patients' Perceived Empathy?
}

Teresa Tomaz , Ana Marta Neves*, Benvinda Barbosa*, Francisco Fachado*, Pedro Fonte**

\section{Resumo}

Introdução: A empatia é uma ferramenta essencial na relação clínica, podendo ser treinada e aferida. Pensa-se que a exposição dos profissionais de saúde a programas de arte possa melhorar a empatia clínica percecionada pelos utentes; porém, desconhece-se qual a sua influência na comunicação com os utentes.

Objetivos: Avaliar a influência dum programa de arte na empatia percecionada pelos utentes relativamente aos profissionais de saúde duma unidade de saúde familiar e verificar a relação entre a empatia percecionada pelos utentes e as características sociodemográficas dos mesmos.

Métodos: Estudo quase-experimental, com avaliação pré e pós-intervenção, conduzido numa unidade de saúde familiar entre janeiro e julho de 2018. Utilizaram-se amostras de conveniência calculadas a partir da população inscrita na unidade de saúde familiar com idade igual ou superior a 18 anos. A intervenção, aplicada aos médicos e enfermeiros, consistiu num programa de arte constituído por três sessões referentes a cinema, literatura e fotografia. Para determinar a influência da intervenção, avaliou-se a empatia pré e pós-intervenção

\begin{abstract}
Introduction: Empathy is an essential component of the doctor-patient relationship which can be measured and trained. The exposure of healthcare practitioners to art-based programs is thought to improve clinical empathy as perceived by patients; however, the exact influence of these programs is unknown.
\end{abstract}

Objectives: To evaluate the influence of an art-based program on patients' perceived empathy of health care providers in a primary care unit and to assess the relationship between patients' sociodemographic characteristics and their perceived empathy of health care providers.

Methods: A quasi-experimental study was conducted in a primary care unit between January and July of 2018, with evaluations made before and after an art-based program was applied. A convenience sample was calculated based on patients aged 18 or older enrolled in the primary care unit. The intervention consisted in an art program directed towards doctors and nurses which included three lectures on cinema, literature and photography. Intervention effectiveness was evaluated by comparing empathy before and after the intervention

\footnotetext{
* USF do Minho, ACeS Cávado I - Braga, ARS Norte.

** USF do Minho, ACeS Cávado I - Braga, Instituto de Investigação em Ciências da Vida e Saúde / Escola de Medicina, Universidade do Minho.
} 
através do questionário "The Consultation and Relational Empathy” e dum questionário sociodemográfico. As associações entre variáveis foram testadas com testes não paramétricos (Mann-Whitney e Kruskal-Wallis) e correlação de Spearman, com um nível de significância de 0,05 .

Resultados: Obtiveram-se 390 e 371 questionários antes e após a intervenção. Verificou-se um aumento estatisticamente significativo da empatia percecionada pelos utentes após a intervenção $(p<0,05)$. As únicas associações estatisticamente significativas verificadas foram com a escolaridade $(p<0,05)$ e situação profissional $(p<0,05)$ no período pré-intervenção.

Discussão: Demonstra-se que um programa de arte aplicado a uma equipa de saúde pode apresentar impacto na empatia percecionada pelos utentes, alertando para a possibilidade de criação futura de espaços de integração artística orientados para o ensino da empatia clínica nos cuidados de saúde primários.

Palavras-chave: “Empatia”, “Relação Médico-Doente”, "Cuidados de saúde primários”, "Arte”, "Competências clínicas de comunicação"

\section{Introdução}

A empatia é um conceito multidimensional que consiste na capacidade para observar as emoções dos outros, para perceber essas emoções e para Ihes dar resposta. ${ }^{1}$ Permite ao profissional de saúde compreender as emoções e sentimentos expressos pelo utente com o objetivo de Ihes dar resposta. Demonstrar empatia implica usar competências que podem ser treinadas e aferidas, traduzindo-se numa componente essencial da aprendizagem clínica. Os estudos realizados encontraram benefícios quanto à satisfação do utente, adesão ao tratamento e à redução do número de queixas., ${ }^{1,2}$

A empatia entende-se como um processo complexo e multidimensional. Alguns autores consideram quatro dimensões empáticas que, em conjunto, beneficiam a prática clínica: emotiva, moral, cognitiva e comportamental. ${ }^{3} \mathrm{~A}$ dimensão emotiva refere-se à capacidade subjetiva de experienciar e partilhar emoções e perspetivas; a moral diz respeito às motivações individuais dos profissionais de saúde; a cognitiva consiste na capacidade objetiva de identificar e compreender as emoções e perspetivas do paciente; a comportamental implica devolver ao paciente esta compreensão. as measured by "The Consultation and Relational Empathy" questionnaire, with additional sociodemographic data simultaneously collected on both occasions. Statistical analysis made use of non-parametric tests (Mann-Whitney e Kruskal-Wallis) and Spearman's correlation test, with a cutoff value of 0.05 for significance.

Results: A total of 390 and 371 questionnaires were obtained before and after the intervention, respectively. A statistically significant improvement of patient's perceived empathy was registered after the intervention $(p<0,05)$. Empathy scores were correlated with education level $(p<0,05)$ and employment status in the pre-intervention period $(p<0,05)$.

Discussion: An art-based program applied to a healthcare team has the potential to improve patient's perceived empathy. These results should prompt the creation of art-based teaching programs directed towards improving clinical empathy in primary care teams.

Keywords: Empathy, Doctor-Patient Relationship, Primary Care, Art, Clinical Communication Skills.

Apesar da generalização de cursos de comunicação clínica, alguns estudos sugerem um declínio dos níveis de empatia nos profissionais de saúde, iniciando-se precocemente no período universitário. ${ }^{4}$ Neste sentido, alguns autores defendem que a participação em programas de arte poderá auxiliar o ensino da empatia nas suas quatro dimensões. ${ }^{5-7} 0$ uso da arte no treino da empatia clínica apresenta várias justificações. As artes visuais (pintura e fotografia) permitem o reconhecimento do comportamento não-verbal do paciente através do estímulo da capacidade de observação do profissional de saúde. ${ }^{8}$ A literatura e o cinema facilitam a identificação e interpretação de situações envolvendo emoções fortes, como a comunicação de más notícias. ${ }^{9}$ A participação direta no processo artístico permite ao profissional explorar e questionar as suas emoções, desenvolvendo a autocrítica e autoreflexão. ${ }^{9}$ Diversas universidades de medicina e enfermagem têm implementado programas de arte nos seus currículos. Atualmente, desconhece-se qual a influência destes programas na empatia demonstrada pelos profissionais de saúde nos cuidados de saúde primários (CSP). 
Este estudo teve como objetivo principal avaliar a influência dum programa de arte na empatia percecionada pelos utentes relativamente aos profissionais de saúde duma unidade de saúde familiar (USF). Adicionalmente, pretendeu-se verificar se existia relação entre a empatia percecionada pelos utentes e as características sociodemográficas dos mesmos.

\section{Métodos}

\section{Desenho do estudo}

Realizou-se um estudo quase-experimental, com avaliação pré e pós-intervenção da empatia percecionada pelos utentes relativamente aos profissionais de saúde duma USF. 0 estudo iniciou-se em março de 2017 com a elaboração do protocolo de investigação, obtendo-se aprovação pela Comissão de Ética da Administração Regional de Saúde do Norte em novembro de 2017. A intervenção consistiu num programa de arte (descrito em detalhe na secção "Intervenção") que foi aplicado aos médicos e enfermeiros da USF. Utilizou-se um questionário composto por duas partes para avaliar a empatia percecionada pelos utentes antes (medida de empatia pré-intervenção) e um mês após (medida de empatia pós-intervenção) a implementação do programa de arte.

A primeira parte do questionário incluiu o instrumento "The Consultation and Relational Empathy" (CARE), validado nos CSP para avaliar a empatia percecionada pelos utentes relativamente aos profissionais de saúde (médicos e enfermeiros). ${ }^{10}$ Trata-se dum questionário anónimo constituído por 10 questões, demorando, em média, 10 minutos a ser preenchido pelo utente após a consulta. As questões são pontuadas numa escala de Likert de 1 (fraco) a 5 (excelente). A medida de empatia é obtida através da soma da pontuação das perguntas de cada questionário, variando de 10 (mínimo) a 50 (máximo). Consideram-se inválidos os questionários com três ou mais respostas "não se aplica". Os questionários com uma ou duas respostas "não se aplica" são considerados válidos, substituindo-se o valor das respostas "não se aplica" pela média das restantes respostas. Utilizou-se o instrumento CARE traduzido para português usado num estudo prévio desenvolvido numa USF em Portugal, tendo-se obtido autorização dos autores deste estudo e dos autores originais. À data da realização deste estudo, este instrumento não se encontra validado em português.
A segunda parte do questionário incluiu questões sobre caraterísticas sociodemográficas: sexo, idade, escolaridade e situação profissional.

Foi entregue um consentimento informado a todos os utentes aquando do preenchimento do questionário e à equipa de saúde que integrou a intervenção realizada para garantir a proteção e confidencialidade. Os custos envolvidos no estudo ficaram a cargo dos autores.

\section{População e amostra do estudo}

A população englobou os utentes com idade igual ou superior a 18 anos inscritos na USF à data da sua realização ( $n=8780)$. Calculou-se uma amostra de conveniência constituída, no mínimo, por 369 utentes com consulta presencial na USF durante o período de aplicação dos questionários, o que corresponde, com um intervalo de confiança de $95 \%$ e uma margem de erro de 5\%, a uma amostra representativa da população da USF. Excluíram-se os utentes que recusassem a participação e que não compreendessem a língua portuguesa.

\section{Aplicação dos questionários}

No momento de efetivação da consulta médica ou de enfermagem, um elemento do secretariado clínico explicava o estudo ao utente caso este integrasse os critérios de inclusão definidos no estudo (utentes com idade igual ou superior a 18 anos que recorressem à USF durante o período de recolha dos questionários, com consulta presencial efetivada para médicos e/ou enfermeiros). O elemento do secretariado clínico excluiria o utente do estudo se não compreendesse a língua portuguesa ou se recusasse participar.

Se o utente aceitasse participar, era instruído a regressar ao secretariado depois da consulta para obter o consentimento informado e o questionário e preenchê-los num espaço definido para o efeito. Os utentes colocavam os questionários preenchidos numa caixa sinalizada e selada.

A aplicação dos questionários ocorreu no período pré-intervenção (janeiro de 2018) e pós-intervenção (julho de 2018). 


\section{Intervenção}

A intervenção consistiu num programa de arte desenvolvido pela investigadora principal do estudo. Este programa decorreu entre março e junho de 2018 e destinou-se à equipa de saúde da USF (seis médicos e seis enfermeiras) e a nove internos de formação específica de medicina geral e familiar. Foram excluídos os secretários clínicos, assistentes operacionais, estudantes de medicina e de enfermagem e outros elementos externos.
O programa de arte consistiu em três sessões versando três formas de arte: cinema, fotografia e literatura. As sessões decorreram em instalações exteriores à USF e tiveram carácter teórico-prático. A descrição das sessões e objetivos respetivos encontram-se detalhadas no quadro 1.

Quadro 1. Descrição e objetivos do programa de arte.

\begin{tabular}{|c|c|c|c|}
\hline Título & Data & Duração & Objetivos \\
\hline $\begin{array}{l}\text { Empatia e } \\
\text { Cinema }\end{array}$ & $10 / 3 / 2018$ & 5 horas & $\begin{array}{l}\text { Objetivos técnicos } \\
\text { - Perceber as formas de transmissão de emoção no cinema (atores, técnica e } \\
\text { argumento) } \\
\text { - Abordar técnicas de comunicação não-verbal no cinema } \\
\text { Objetivos clínicos } \\
\text { - Perceber a importância da entrevista clínica na relação entre o profissional de saúde } \\
\text { e o paciente } \\
\text { - Entender a diferença entre entrevista centrada no paciente e no profissional de saúde } \\
\text { - Identificar barreiras na transmissão de empatia } \\
\text { - Compreender a importância da escuta ativa } \\
\text { - Conhecer técnicas de transmissão de empatia }\end{array}$ \\
\hline $\begin{array}{l}\text { Empatia e } \\
\text { Fotografia }\end{array}$ & $5 / 5 / 2018$ & 8 horas & $\begin{array}{l}\text { Objetivos técnicos } \\
\text { - Explorar a câmara fotográfica e as suas capacidades técnicas } \\
\text { - Abordar a linguagem da composição fotográfica } \\
\text { - Analisar a técnica da fotografia de rua e de retrato } \\
\text { - Perceber as formas de transmissão de emoção na fotografia } \\
\text { Objetivos clínicos } \\
\text { - Compreender os pressupostos da construção não-verbal da empatia } \\
\text { - Perceber a importância da observação atenta na identificação dos comportamentos } \\
\text { não-verbais e padrões comportamentais }\end{array}$ \\
\hline $\begin{array}{l}\text { Empatia e } \\
\text { Literatura }\end{array}$ & $16 / 6 / 2018$ & 5 horas & $\begin{array}{l}\text { Objetivos técnicos } \\
\text { - Abordar o uso de narrativas literárias e não literárias } \\
\text { - Abordar o conceito de medicina baseada na narrativa } \\
\text { Objetivos clínicos } \\
\text { - Conhecer competências empáticas de resposta a situações emocionais fortes } \\
\text { - Identificar dificuldades e erros na comunicação de más notícias } \\
\text { - Abordar estratégias empáticas perante um paciente "difícil" } \\
\text { - Compreender a importância clínica do auto-conhecimento }\end{array}$ \\
\hline
\end{tabular}

A primeira sessão focou-se no cinema. Foi utilizada uma apresentação em formato Powerpoint ${ }^{\circledR}$ para abordar conceitos teóricos relativos às técnicas cinematográficas e à sua utilização na transmissão de empatia e de emoções fortes no cinema. Para a componente prática, recorreu-se a um ambiente experiencial psicodramático com recurso a roleplay com vista à interpretação de algumas das situações retratadas nas cenas de filmes visualizadas na sessão teórica. A equipa de saúde participou em situações de roleplay e debateu a condução das diversas entrevistas clínicas, propondo estratégias para melhorar a empatia transmitida. Como exercício prático, os participantes identificaram os principais erros de comunicação em diversas cenas. No final da sessão foi entregue uma lista de filmes sugeridos e um guia de bolso de transmissão de empatia elaborado pelas autoras do estudo.

A segunda sessão incidiu sobre a fotografia. Previamente foi solicitado aos participantes que levassem individualmente uma câmara fotográfica ou um telemóvel com câmara. A sessão teórica foi dividida em duas partes: a primeira correspondeu a um curso breve de introdução à fotografia, onde foram abordados conceitos gerais sobre técnicas fotográficas. A segunda parte incidiu sobre a interpretação fotográfica, recorrendo-se a vários projetos de fotografia e trabalhos de fotógrafos profissionais como exemplos. A componente prática decorreu no exterior, 
onde os participantes tiraram fotografias de rua e de retrato. Posteriormente, os participantes formaram dois grupos e utilizaram máquinas fotográficas descartáveis, tendo sido solicitado que imaginassem o que gostariam de transmitir com as suas fotografias.

A terceira sessão abordou a literatura. Esta sessão foi dinamizada através dum grupo de discussão, em que a investigadora principal participou como moderadora. Previamente à sessão foi entregue à equipa um documento intitulado "Empatia e Literatura", consistindo numa análise a diferentes textos e obras literárias de ficção e não-ficção. Foram abordados e discutidos conceitos teóricos relativamente a situações de comunicação de más notícias, transmissão de empatia perante um paciente agressivo e conceitos relativos a dolência e doença através da análise das obras selecionadas.

\section{Análise estatística}

Para a análise estatística utilizaram-se os programas Microsoft Excel ${ }^{\circledR}$ e Statistical Package for the Social Sciences (SPSS), versão $25^{\circledR}$.

Numa primeira etapa, foi efetuada a análise descritiva. Para as variáveis categóricas (nominais e ordinais) foram determinadas as frequências absolutas e relativas e para as numéricas determinaram-se as medidas de localização central e dispersão. Foram apresentadas as medianas e amplitude interquartil para as distribuições assimétricas.

Para determinar se as amostras eram estatisticamente diferentes, aplicou-se o teste qui-quadrado às proporções das variáveis demográficas sexo, situação profis- sional e escolaridade no período pré e pós-intervenção e o teste t-student para amostras independentes para a variável idade.

Visando testar a associação da medida de empatia com a idade, utilizou-se o teste de correlação de Spearman. Utilizaram-se os testes não paramétricos de Mann-Whitney e Kruskal-Wallis para investigar a associação entre as restantes variáveis sociodemográficas e a medida de empatia. Através da aplicação do teste de Mann-Whitney, realizou-se uma análise comparativa dos resultados obtidos antes e após a intervenção. Para todos os testes estatísticos, o nível de significância foi estabelecido em $5 \%(p<0,05)$.

Considerou-se que a eficácia da intervenção traduzirse-ia num aumento da mediana da medida de empatia com um valor estatisticamente significativo.

\section{Resultados}

Foram obtidos 390 questionários válidos no período pré-intervenção e 371 no período pós-intervenção. No período pré-intervenção, consideraram-se 12 questionários inválidos e no período pós-intervenção 23 questionários inválidos por conterem mais de duas perguntas com a resposta "não sabe/não responde" e questionários sociodemográficos em branco.

O quadro 2 descreve as caraterísticas sociodemográficas das amostras.

Quadro 2. Caraterísticas sociodemográficas da amostra pré-intervenção e pós-intervenção.

\begin{tabular}{|c|c|c|c|c|}
\hline \multicolumn{2}{|c|}{ Variáveis sociodemográficas } & Pré-intervenção n (\%) & Pós-intervenção n (\%) & Teste qui-quadrado \\
\hline \multirow{2}{*}{ Sexo } & Feminino & $278(71,3)$ & $249(67,1)$ & \multirow{2}{*}{$1,55(p=0,213)^{*}$} \\
\hline & Masculino & $112(28,7)$ & $122(32,9)$ & \\
\hline \multirow{4}{*}{ Escolaridade } & Até ao $4 .^{\circ}$ ano & $66(16,9)$ & $63(17)$ & \multirow{4}{*}{$2,57(p=0,462)^{*}$} \\
\hline & $5 .^{\circ}$ ao $9 .^{\circ}$ ano & $93(23,8)$ & $74(19,9)$ & \\
\hline & $10 .^{\circ}$ ao $12 .^{\circ}$ ano & $103(26,4)$ & $114(30,7)$ & \\
\hline & Superior ao $12 .^{\circ}$ ano & $128(32,8)$ & $120(32,3)$ & \\
\hline \multirow{4}{*}{ Situação profissional } & Estudante & $23(5,9)$ & $25(6,7)$ & \multirow{4}{*}{$3,718(p=0,445)^{*}$} \\
\hline & Ativo & $233(59,7)$ & $222(59,8)$ & \\
\hline & Desempregado & $36(9,2)$ & $45(12,1)$ & \\
\hline & Reformado & $97(24,9)$ & $79(21,3)$ & \\
\hline \multirow{2}{*}{ Idade } & & Média pré-intervenção & Média pós-intervenção & Teste t-student \\
\hline & & 46,7 & 42,4 & $t=0,581(p=0,948)^{+}$ \\
\hline
\end{tabular}

Legenda: * Teste de Qui-Quadrado; ${ }^{+}$Teste t-student para amostras independentes 
No período pré-intervenção, a média de idades foi de 46,7 anos. A média da medida de empatia foi de 42,4 pontos, com um desvio padrão de 8,4. A mediana da medida de empatia obtida foi de 45 pontos.

Foi aplicado o teste de qui-quadrado, conforme descrito na metodologia, tendo-se obtido valores de $p>0,05$, aceitando-se a hipótese nula, isto é, as amostras no período pré e pós-intervenção são estatisticamente semelhantes quanto ao sexo, escolaridade e situação profissional. Foi aplicado o teste t-student para amostras independentes para a variável idade, tendo-se obtido também valores de $p>0,05$, concluindo-se que as amostras são estatisticamente semelhantes quanto à idade.

O teste de correlação de Spearman foi aplicado para investigar a associação entre as variáveis idade e empatia, dada a distribuição assimétrica da amostra. Os resultados desta correlação indicaram que houve uma associação muto fraca entre a idade e empatia, com significância estatística ( $r s=-0,18 ; p<0,05)$. Conforme se constata pela análise do quadro 3 , não se verificou uma associação estatisticamente significativa entre as variáveis sexo e medida de empatia $(U=14097$; $z=-1,490$; p>0,05; r=-0,08); porém, verificou-se uma relação estatisticamente significativa entre a medida de empatia e as variáveis escolaridade $(H=22,602 ; p<0,05)$ e situação profissional $(H=16,173 ; p<0,05)$. Constatou-se que os utentes com maior escolaridade atribuem uma medida de empatia mais elevada comparativamente aos de escolaridade inferior. Verificou-se também que os utentes profissionalmente ativos ou estudantes atribuem uma medida de empatia superior relativamente aos desempregados e reformados.

No período pós-intervenção, a média de idades foi de 46,0 anos. A média da medida de empatia foi de 47,2 pontos, com um desvio padrão de 4,2. A mediana da medida de empatia obtida foi de 50 pontos. À semeIhança do período pré-intervenção, os resultados do teste de correlação de Spearman indicaram uma relação muito fraca entre a medida de empatia e a idade de forma estatisticamente significativa ( $r s=-0,158 ; p<0,05$ ), e não se verificou uma relação estatisticamente significativa entre a medida de empatia e o sexo $(U=13444$; $z=-1,947 ; p>0,05 ; r=-0,10)$. Porém, também não se verificou uma relação estatisticamente significativa entre as restantes variáveis sociodemográficas com a medida de empatia (quadro 3).

Quadro 3. Associação entre os níveis de empatia e as variáveis estudadas.

\begin{tabular}{|c|c|c|c|c|c|c|c|}
\hline \multirow{2}{*}{\multicolumn{2}{|c|}{ Variáveis sociodemográficas }} & \multicolumn{3}{|c|}{ Pré-intervenção } & \multicolumn{3}{|c|}{ Pós-intervenção } \\
\hline & & Mediana ME & AIQ & $\mathrm{p}$ & Mediana ME & AIQ & $\mathrm{p}$ \\
\hline \multirow{2}{*}{ Sexo } & Feminino & 43 & 12 & \multirow{2}{*}{$0,136^{*}$} & 49 & 4 & \multirow{2}{*}{$0,052^{*}$} \\
\hline & Masculino & 46 & 11 & & 50 & 7 & \\
\hline \multirow{4}{*}{ Escolaridade } & Até ao $4 .^{\circ}$ ano & 38 & 16 & \multirow{4}{*}{$0,000^{+}$} & 48 & 6 & \multirow{4}{*}{$0,333^{+}$} \\
\hline & $5 .^{\circ}$ ao $9 .^{\circ}$ ano & 43 & 17 & & 50 & 7 & \\
\hline & $10 .^{\circ}$ ao $12 .^{\circ}$ ano & 47 & 10 & & 50 & 4 & \\
\hline & Superior ao $12 .^{\circ}$ ano & 48 & 10 & & 50 & 2 & \\
\hline \multirow{4}{*}{ Situação profissional } & Estudante & 47 & 8 & \multirow{4}{*}{$0,001^{+}$} & 50 & 2 & \multirow{4}{*}{$0,206^{+}$} \\
\hline & Ativo & 47 & 10 & & 50 & 4 & \\
\hline & Desempregado & 41 & 13 & & 50 & 7 & \\
\hline & Reformado & 41 & 16 & & 49 & 8 & \\
\hline
\end{tabular}

Legenda: * Teste de Mann-Whitney; ${ }^{+}$Teste de Kruskal-Wallis; ME - medida de empatia; AIQ - amplitude interquartil 
Globalmente, a mediana da medida de empatia aumentou depois da intervenção, sendo a diferença estatisticamente significativa (45 vs 50 pontos; $U=50765$; $z=-7,342$; $p<0,05 ; r=-0,27$ ) (quadro 4).

Quando foi realizada a análise da mediana de cada pergunta do questionário CARE (mínimo de um ponto e máximo de cinco pontos), verificou-se que no período pré-intervenção a mediana atribuída a todas as questões foi de cinco pontos, excetuando as questões número seis (“Mostrar preocupação e compaixão ao mostrar-se ge- nuinamente preocupado(a), interagindo consigo ao nível humano") e dez ("Fazer um plano de ação consigo, ao discutir consigo as opções, envolvendo-o nas decisões na medida em que você quer estar envolvido") que apresentaram uma mediana de quatro pontos. No período pós-intervenção, a mediana das pontuações atribuídas a cada questão foi de cinco pontos; esta diferença apresentou significância estatística aplicando-se o teste de Mann-Whitney $(p<0,05)$ (quadro 4).

Quadro 4. Mediana da empatia global e por questão antes e após a intervenção

\begin{tabular}{|c|c|c|c|c|c|c|}
\hline \multirow{2}{*}{\multicolumn{2}{|c|}{ Medida de empatia }} & \multicolumn{2}{|c|}{ Pré-intervenção } & \multicolumn{2}{|c|}{ Pós-intervenção } & \multirow{2}{*}{ p-value* } \\
\hline & & Mediana & AIQ & Mediana & AIQ & \\
\hline \multicolumn{2}{|l|}{ Global } & 45 & 12,1 & 50 & 5 & 0,00 \\
\hline \multirow{10}{*}{ Por questão } & Questão 1 & 5 & 1 & 5 & 0 & 0,00 \\
\hline & Questão 2 & 5 & 1 & 5 & 1 & 0,00 \\
\hline & Questão 3 & 5 & 1 & 5 & 1 & 0,00 \\
\hline & Questão 4 & 5 & 1 & 5 & 1 & 0,00 \\
\hline & Questão 5 & 5 & 1 & 5 & 1 & 0,00 \\
\hline & Questão 6 & 4 & 1,25 & 5 & 1 & 0,00 \\
\hline & Questão 7 & 5 & 1 & 5 & 1 & 0,00 \\
\hline & Questão 8 & 5 & 1 & 5 & 0 & 0,00 \\
\hline & Questão 9 & 5 & 1 & 5 & 0,5 & 0,00 \\
\hline & Questão 10 & 4 & 1 & 5 & 1 & 0,00 \\
\hline
\end{tabular}

Legenda: * Teste de Mann-Whitney; AIQ - amplitude interquartil

\section{Discussão}

A implementação de programas de arte destinados a profissionais de saúde pré e pós-graduados surge na tentativa de dar resposta ao declínio dos níveis de empatia verificados ao longo do percurso académico e clínico. Porém, a dificuldade na concetualização e na avaliação da eficácia destes programas tem limitado a sua implementação. Além disso, considerou-se que estes programas apenas poderiam beneficiar as dimensões emotiva e moral da empatia, deixando de parte as cognitiva e comportamental, fundamentais à prática clínica.

Este estudo pretendeu perceber qual a influência dum programa de arte na empatia percecionada pelos utentes duma USF. Antes da intervenção, a média da medida de empatia obtida foi de 42,4 pontos, traduzindo um valor semelhante ao estudo realizado por Macedo et al em 2011 numa USF em Portugal. ${ }^{11}$ Após o programa, verificou-se um aumento da medida de empatia percecionada pelos utentes relativamente aos profissionais de saúde. Parece, assim, que um programa de arte pode influenciar a empatia demonstrada pelos profissionais de saúde.

Quando analisada cada questão do questionário CARE, verificou-se que os utentes atribuíram uma pontuação inferior nas questões número seis e dez no período préintervenção, constatando-se uma melhoria estatisticamente significativa no período pós-intervenção. Sabe-se que as questões número um e seis do questionário CARE se relacionam com a dimensão emotiva da empatia, e as restantes com as dimensões comportamental e cognitiva. ${ }^{10}$ Assim, parece que um programa de arte pode ter impacto nas quatro dimensões empáticas. 
Este estudo demonstrou uma associação entre a medida de empatia e as variáveis sociodemográficas escolaridade e situação profissional no período pré-intervenção. Assim, verificou-se que os níveis de empatia atribuídos pelos utentes aumentavam de acordo com o aumento da escolaridade. Verificou-se também que os estudantes e utentes profissionalmente ativos atribuíam pontuações mais elevadas nos questionários de medida de empatia comparativamente aos reformados e desempregados. Uma explicação que pode justificar este resultado é o facto de os profissionais de saúde se identificarem mais com indivíduos com maior escolaridade e com os estudantes e ativos profissionalmente. No estudo realizado por Macedo et al, verificou-se apenas uma associação semelhante com a escolaridade. ${ }^{11}$

A associação encontrada neste estudo foi estatisticamente significativa apenas no período pré-intervenção. Este facto poderá ser explicado pelo aumento da medida da empatia no período pós-intervenção, atenuando as diferenças encontradas entre os diferentes grupos sociodemográficos.

Importa referir que a metodologia usada apresenta limitações, nomeadamente a inexistência dum grupo de controlo não exposto à intervenção e a utilização duma amostra de conveniência, não tendo sido possível utilizar a mesma amostra antes e após a intervenção. Apesar de as amostras não serem iguais, tentou-se ultrapassar esta limitação através da aplicação do teste de qui-quadrado, que demonstrou que ambas eram estatisticamente semelhantes. Adicionalmente, o questionário CARE não está validado na língua portuguesa. Importa ainda referir que foi avaliado o efeito do programa de arte num curto espaço de tempo após a intervenção ter sido concluída, desconhecendo-se o seu efeito a longo prazo.

Apesar do reconhecimento da importância da empatia na entrevista clínica, os programas formativos universitários e especializados ainda não dispõem de ferramentas de ensino estruturado de competências de comunicação em contexto clínico suficientes e universalmente difundidas. Espera-se, com este estudo, alertar para o benefício da criação futura de espaços de integração artística orientados para o ensino da empatia clínica. Seria interessante estudar o eventual impacto destes programas no burnout dos profissionais de saúde e, futuramente, replicar este estudo com a utilização dum grupo controlo, colmatando as limitações descritas, bem como estudar o impacto da intervenção a longo prazo. Assim, os autores concluem que estes programas poderão ser adjuvantes importantes no ensino da empatia médica nos CSP.

\section{Agradecimentos}

Os autores agradecem aos responsáveis das instalações onde decorreram as sessões do programa de arte. Agradecem ainda ao Dr. António Macedo pela disponibilização do questionário CARE traduzido em português e a toda a equipa de saúde que aceitou participar no projeto.

\section{Referências Bibliográficas}

1. Cardoso RM. Competências Clínicas de Comunicação. $1^{\text {st }}$ ed. Porto: Unidade de Psicologia Médica da Faculdade de Medicina da Universidade do Porto; 2012.

2. Derksen F, Bensing J, Lagro-Janssen A. Effectiveness of empathy in general practice: a systematic review. $\mathrm{Br} J$ Gen Pract. 2013 Jan 1;63(606):e76-84.

3. Stepien KA, Baernstein A. Educating for empathy: A review. J Gen Intern Med. 2006;21(5):524-30.

4. Kelm Z, Womer J, Walter JK, Feudtner C. Interventions to cultivate physician empathy: a systematic review. BMC Med Educ. 2014;14(1):219.

5. Larson EB, Yao X. Clinical empathy as emotional labor in the patient-physician relationship. JAMA. 2005;293(9):1100-6.

6. Potash JS, Chen JY, Lam CL, Chau VT. Art-making in a family medicine clerkship: How does it affect medical student empathy? BMC Med Educ. 2014;14(1):1-9.

7. Graham J, Benson LM, Swanson J, Potyk D, Daratha K, Roberts K. Medical Humanities Coursework Is Associated with Greater Measured Empathy in Medical Students. Am J Med. 2016;129(12):1334-7.

8. Elder NC, Tobias B, Lucero-Criswell A, Goldenhar L. The art of observation: Impact of a family medicine and art museum partnership on student education. Fam Med. 2006;38(6):393-8.

9. Perry M, Maffulli N, Willson S, Morrissey D. The effectiveness of arts-based interventions in medical education: $A$ literature review. Med Educ. 2011;45(2):141-8.

10. Mercer SW, McConnachie A, Maxwell M, Heaney D, Watt GCM. Relevance and practical use of the Consultation and Relational Empathy (CARE) Measure in general practice. Fam Pract. 2005;22(3):328-34.

11. Macedo A, Cavadas LF, Sousa M, Pires P, Santos JA, Machado A. Empathy in Family Medicine. Rev Port Clin Geral. 2011;27:527-32.

\section{Conflito de Interesses}

Os autores declaram não ter conflito de interesses.

\section{Endereço para Correspondência}

Teresa Tomaz: tomaz.teresa@gmail.com

Ana Marta Neves: ana.marta2688@gmail.com

Benvinda Barbosa: benvindabarbosa25@gmail.com

Francisco Fachado: franciscofachado.usfdominho@gmail.com

Pedro Fonte: fonte.pedro.a@gmail.com 